\title{
Addressing a cavity with patterns at ultra-wideband detune
}

\author{
Xiaoming Wei, ${ }^{1}$ Yiqing $\mathrm{Xu},{ }^{1}$ Sisi Tan, ${ }^{1}$ Shanhui $\mathrm{Xu},{ }^{2}$ Zhongmin Yang, ${ }^{2, *}$ \\ Kevin K. Tsia, ${ }^{1}$ and Kenneth K. Y. Wong, ${ }^{1, *}$ \\ ${ }^{I}$ Department of Electrical and Electronic Engineering, The University of Hong Kong, Pokfulam Road, Hong Kong. \\ ${ }^{2}$ Institute of Optical Communication Materials and State Key Laboratory of Luminescent Materials and Devices, South China University of \\ Technology, Guangzhou 510641, P. R. China \\ *E-mail: yangzm@scut.edu.cn; kywong@eee.hku.hk
}

\begin{abstract}
We demonstrate an amplified fiber ring cavity at telecommunication window addressed by optical pattern at $1.0 \mu \mathrm{m}$. A storage time longer than $38 \mu$ s and an ultra-wideband wavelength conversion of $\sim 500 \mathrm{~nm}$ have been obtained.

(C)2014 Optical Society of America

OCIS codes: (190.4370) Nonlinear optics, fibers; (190.4223) Nonlinear wave mixing
\end{abstract}

\section{Introduction}

While dissipative soliton (DS) in fiber laser has already found widespread applications [1], the temporal cavity soliton (TCS, an extended terminology of spatial cavity soliton [2]) in a coherently-driven fiber cavity has been observed recently [3]. The TCS cavities were driven by an external continuous-wave (CW) laser with ultra-narrow linewidth $(\mathrm{kHz})$ instead of a conventional optical amplifier, which compensates the cavity loss. It has been shown that TCS can be excited with an external pulse laser at a nearby wavelength of the fiber cavity [3,4], or even be spontaneously generated without external stimulation in microresonators [5]. As a promising candidate for storing bits in an all-optical buffer, TCS has showcased its multiple telecommunication functions, e.g. all-optical storage and wavelength conversion [3]. This stabilized fiber cavity, however, has to be driven with an ultra-narrow linewidth CW laser at around $1550 \mathrm{~nm}$ for coherent gain, and stimulated by an external pulse laser at $\sim 1530 \mathrm{~nm}$ for TCS generation. The operation range as a wavelength convertor, in addition, is limited to about $20 \mathrm{~nm}$. As far as the simplicity and cost are concerned, it would be very beneficial to develop a compact and cost-effective design with a larger wavelength conversion range. Here, we demonstrate an all-fiber oscillating cavity at $1.55 \mu \mathrm{m}$ driven by an off-the-shelf laser diode (LD), which has a spectral width of $<0.2 \mathrm{~nm}$ centered at $980 \mathrm{~nm}$. The wavelength conversion and optical storage are realized by addressing the all-fiber cavity with a pulse laser at $1.0 \mu \mathrm{m}$. Associated with an ultra-wideband wavelength conversion of $500 \mathrm{~nm}$, this scheme shows promising features over other all-optical delay lines [6].

\section{Experimental setup and results}

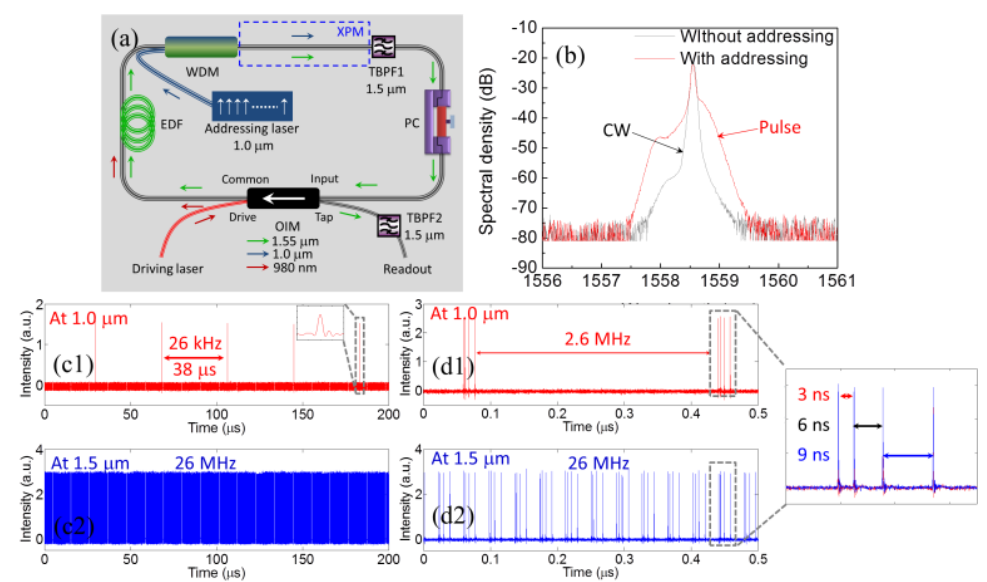

Fig. 1. (a) The experimental setup of the oscillating fiber cavity. (b) The optical spectra of the oscillating fiber cavity with and without addressing beam. (c1) The addressing pulse train at $1.0 \mu \mathrm{m}$ with an addressing period of $38 \mu \mathrm{s}$. (c2) The excited pulse train of the fiber cavity at $1.55 \mu \mathrm{m}$ with a pulsing period of $\sim 38.1 \mathrm{~ns}$, i.e. the round trip time (RTT). (d1) The addressing pattern with four 1-bit at $1.0 \mu \mathrm{m}$. (d2) The excited pattern at $1.55 \mu \mathrm{m}$. Inset: the overlapping of the addressing and excited patterns.

The all-fiber cavity, as shown in Fig. 1(a), incorporates the pattern excitation via cross-phase modulation (XPM) and sustaining through an artificial saturable absorber, which was implemented by a fiber-based multi-functional optical 
integrated module (OIM) [7]. Different from Ref. 3, the fiber cavity in our scheme was driven by a 980-nm LD with a maximum output power of $\sim 300 \mathrm{~mW}$ and a spectral width of $<0.2 \mathrm{~nm}$. The $980-\mathrm{nm}$ laser beam, reflected into the common port of OIM via its internal dichroic mirror, would be completely absorbed by a piece of 1-m erbium-doped fiber (EDF) and thus compensated the cavity loss. The output from the EDF would be combined with the addressing beam from an external pulse laser at $1.0 \mu \mathrm{m}$ through a fiber-based WDM coupler. The combined lightwave at $1.55 \mu \mathrm{m}$ and addressing beam at $1.0 \mu \mathrm{m}$ would interact with each other through XPM in a standard single-mode fiber $(\sim 3 \mathrm{~m}$ in length) between the WDM coupler and a tunable bandpass filter (TBPF1, blocked the 1.0- $\mu \mathrm{m}$ component). Here, the fiber cavity would be deliberately set to only emit $\mathrm{CW}$ lightwave at $1.55 \mu \mathrm{m}$ without the addressing beam, and then the $1.55-\mu \mathrm{m}$ CW lightwave would be "modulated", via XPM, to be the pulse pattern the same as that of the addressing beam being introduced, as shown in Fig. 1(b).

First, we examined the case that the addressing optical pattern only contained one 1-bit, as shown in the inset of the Fig. 1(c1). With different addressing periods, which were much longer than the round trip time (RTT) of the fiber cavity, the oscillation of the fiber cavity could evolve from $\mathrm{CW}$ to pulse pattern exactly the same as that of the addressing beam. Fig. 1(c) shows the results of the addressing case when addressing period was 1000 RTTs. As can be observed, the addressing pattern at $1.0 \mu \mathrm{m}$ excited the fiber cavity every $38 \mu$ s, i.e. $26 \mathrm{kHz}$. The fiber cavity delivered a pulsed-pattern train at its fundamental repetition rate $(26.2 \mathrm{MHz})$, and no decay between neighboring addressing pulses was observed. With the autocorrelator, the pulsewidth of the excited pulse in the fiber cavity was measured to be $\sim 14 \mathrm{ps,} \mathrm{which} \mathrm{is} \mathrm{comparable} \mathrm{with} \mathrm{that} \mathrm{of} \mathrm{the} \mathrm{addressing} \mathrm{pulse,} \mathrm{i.e.} 16 \mathrm{ps}$. The slight discrepancy can be attributed to pulse compression effect of the artificial saturable absorber. The timing jitter of the excited pulse was measured to be $<2$ ps. Fig. 1(d) shows the case addressed with four 1-bit pattern. The bit separation between the four 1-bit addressing pulses is about $3 \mathrm{~ns}, 6 \mathrm{~ns}$ and $9 \mathrm{~ns}$, respectively, as shown in the inset of Fig. 1(d). By overlapping both the addressing and excited patterns together, it shows a very good consistency. It is noted that the data bits stored in an all-optical buffer can be interfered by the repulsive interaction between solitons [4]. To study the highest bit-rate (or smallest temporal separation between bits) that can be stored by this fiber cavity, we varied the temporal separation between bits and evaluated the addressing results by real-time oscilloscope and autocorrelator with a temporal resolution of < $10 \mathrm{fs}$. It was found that the fiber cavity delivered a good consistency between the addressing and excited patterns when the bit separation was longer than $20 \mathrm{ps}$, while the repulsive interaction for a shorter separation became much stronger [1]. This suggests that the fiber cavity can support a bit-rate of $50 \mathrm{Gbit} / \mathrm{s}$.

\section{Conclusion}

In conclusion, we have demonstrated the dynamics of a fiber ring cavity at $1.55 \mu \mathrm{m}$ addressed by external optical pattern at $1.0 \mu \mathrm{m}$, and shown that it can be a fruitful platform for studying the nonlinear dissipative dynamics. Addressing by different optical patterns, the amplified fiber ring cavity exhibited a buffering time longer than $38 \mu \mathrm{s}$, at an equivalent bit-rate of $50 \mathrm{Gbit} / \mathrm{s}$. With an all-fiber configuration and simple driving-source requirement, together with an effective wavelength conversion with a working range of $\sim 500 \mathrm{~nm}$, this scheme can be a promising candidate for optical buffer over other all-optical delay lines.

\section{Acknowledgment}

This work was partially supported by grant from the Research Grants Council of the Hong Kong Special Administrative Region, China (Project No. HKU 17208414, HKU 7172/12E, HKU 717510E, HKU 717911E, HKU 720112E) and University Development Fund of HKU.

\section{References}

[1] P. Grelu and N. Akhmediev, "Dissipative solitons for mode-locked lasers," Nat. Photonics 6, 84-92 (2012).

[2] S. Barland, J. R. Tredicce, M. Brambilla, L. A. Lugiato, S. Balle, M. Giudici, T. Maggipinto, L. Spinelli, G. Tissoni, T. Knödl, M. Miller, and R. Jäger, "Cavity solitons as pixels in semiconductor microcavities," Nature 419, 699-702 (2002).

[3] F. Leo, S. Coen, P. Kockaertl, S.-P. Gorza, P. Emplit, and M. Haelterman, "Temporal cavity solitons in one-dimensional kerr media as bits in an all-optical buffer," Nat. Photonics 4, 471-476 (2010).

[4] J. K. Jang, M. Erkintalo, S. G. Murdoch, and S. Coen, "Ultraweak long-range interactions of solitons observed over astronomical distances," Nat. Photonics 7, 657-663 (2013).

[5] T. Herr, V. Brasch, J. D. Jost, C. Y. Wang, N. M. Kondratiev, M. L. Gorodetsky, T. J. Kippenberg, "Temporal solitons in optical microresonators," Nat. Photonics 8, 145-152 (2014).

[6] N. Alic, J. R. Windmiller, J. B. Coles, and S. Radic, “Two-Pump Parametric Optical Delays,” IEEE J. Sel. Top. Quantum Electron. 14, 681-690 (2008).

[7] X. Wei, J. Xu, Y. Xu, L. Yu, J. Xu, B. Li, A. K. S. Lau, X. Wang, C. Zhang, K. K. Tsia, and K. K. Y. Wong, ”Breathing laser as an inertia-free swept source for high-quality ultrafast optical bioimaging," Opt. Lett. 39, 6593-6596 (2014). 